\title{
The putative tumor suppressor, miR-199a, regulated by Snail, modulates clear cell renal cell carcinoma aggressiveness by repressing ROCKI
}

This article was published in the following Dove Press journal:

OncoTargets and Therapy

\author{
Xiao Zhang' \\ Peng $\mathrm{Li}^{1}$ \\ Zhen Ding' \\ Huili Wang' \\ Junye Wang' \\ Lei Han' \\ Shangwei Ding² \\ 'Department of Oncology, Affiliated \\ Hospital of Jining Medical University, \\ Jining, Shandong, ${ }^{2}$ Department of \\ Ultrasound, Dongguan People's \\ Hospital Affiliated to Southern \\ Medical University, Dongguan, \\ Guangdong, China
}

Correspondence: Lei Han Department of Oncology, Affiliated Hospital of Jining Medical University, Number 89 Guhuai Road, Jining 272029,

Shandong, China

Tel +865372903399

$\mathrm{Fax}+865372213030$

Email hanleixxf@I26.com

\section{Shangwei Ding}

Department of Ultrasound, Dongguan

People's Hospital Affiliated to Southern Medical University, Number 3, New

Valley Chung Wan Road South, Wanjiang

District, Dongguan 523059, Guangdong,

China

Tel +8676928637333

Fax +86 76922222353

Email dingshangwei_1980@I63.com
Background: Aberrant expression of miR-199a has been frequently reported in cancer studies; however, its role in renal cell carcinoma (RCC) has not been examined in detail.

Results: Here, we showed that miR-199a was downregulated in RCC and associated with poor prognostic phenotype. Using luciferase and western blot assays we identified that Rhoassociated coiled coil-containing protein kinases 1 (ROCK1) was a direct target gene for miR-199a. miR-199a regulated proliferation, invasion, and apoptosis of clear cell renal cell carcinoma (ccRCC) cells by modulating ROCK1 expression. Interestingly, we also found that miR-199a was modulated by snail in ccRCC cells. Snail elevated ROCK1 expression by repressing miR-199a activity.

Conclusion: Altogether, our results identify a crucial tumor suppressive role of miR-199a in the progression of ccRCC and suggest that miR-199a might be an anticancer therapeutic target for ccRCC patients.

Keywords: clear cell renal cell carcinoma, miR-199a, ROCK1, Snail, A498

\section{Introduction}

Renal cell carcinoma (RCC), accounting for $90 \%-95 \%$ of all renal tumors in adults, ${ }^{1}$ comprises a heterogeneous group of epithelial neoplasms with diverse biologic potential and variable clinical outcomes. ${ }^{2}$ Compared with the other two major subtypes, chromophobe and papillary RCC, clear cell renal cell carcinoma (ccRCC) is the most common and associated with worse prognosis. ${ }^{3}$ Radical or partial nephrectomy remains the mainstay of treatment for localized RCC, but alone it provides limited benefit in patients with aggressive disease. ${ }^{4}$ In addition, RCC is resistant to chemotherapy and immunotherapy in most cases. ${ }^{5}$ Recent years have seen a rapid development of molecular targeted therapy in the treatment of advanced RCC. However, most of the treatments are not curative, and side effects associated with targeted therapy cannot be ignored. ${ }^{6-8}$ Thus, new correlative markers and therapeutic agents for RCC are urgently needed.

Rho-associated coiled coil-containing protein kinase 1 (ROCK1) is a member of the Rho-associated serine/threonine kinase family. ROCK1 controls a wide variety of ubiquitous biological processes including the acquisition of unlimited proliferation potential, survival and evasion from apoptosis, tissue invasion, differentiation, gene expression and in particular, regulation of cell detachment, cell movement and establishment of metastasis..$^{9,10}$

Snail is a transcriptional inhibitory protein, which usually promotes cancer growth and invasion. ${ }^{11}$ It has been reported that Snail is highly expressed in ccRCC 
and is positively associated with tumor stage but negatively related to patients' prognosis. ${ }^{12}$ Recently, it has been documented that Snail can exert its function through regulating microRNAs (miRNAs) expression. ${ }^{13}$ However, the role of Snail and miRNAs in ccRCC is still poorly investigated.

miRNAs, which are single-stranded 19- to 25-nucleotide short RNAs, function as negative posttranscriptional regulators of target genes. ${ }^{14-16}$ Recent evidence suggests that miRNAs are aberrantly expressed during the development and/or progression of a variety of human cancers. ${ }^{17,18}$ Recently, several studies have showed that miR-199a is dysregulated and represses tumor progression in chondrosarcoma ${ }^{19}$ and hepatocellular ${ }^{20}$ and ovarian cancer. ${ }^{21}$ In one study, increased miR-199a was observed in gastric cancer, ${ }^{22}$ suggesting its function as an oncogene. However, biological functions of miR-199a in the control of ccRCC aggressiveness have not been well characterized. Here, we sought to investigate the potential role of miR-199a in the development and progression of ccRCC.

\section{Materials and methods Cell lines and patient samples}

A498 cell line was purchased from the Institute of Biochemistry and Cell Biology of the Chinese Academy of Sciences (Shanghai, China) and cultured in DMEM supplemented with 10\% fetal bovine serum (FBS), $100 \mathrm{U} / \mathrm{mL}$ penicillin and $100 \mathrm{mg} / \mathrm{mL}$ streptomycin in humidified air at $37^{\circ} \mathrm{C}$ with $5 \% \mathrm{CO}_{2}$. ccRCC and adjacent normal tissue samples were obtained with informed consent under Institutional Review Board-approved protocols. ccRCC tissue samples from 98 patients were collected from the Affiliated Hospital of Jining Medical University, Jining, Shandong, China. The study was approved by the Institutional Review Board of Affiliated Hospital of Jining Medical University, and written informed consent was obtained from each patient.

\section{Quantitative real-time PCR}

Total RNA was extracted with TRIzol reagent (Thermo Fisher Scientific, Waltham, MA, USA). For the detection of miR199a, reverse transcription and quantitative real-time PCR (qRT-PCR) analysis were carried out with the use of SYBR Premix Ex Taq (Takara, Kusatsu, Japan). The primers used for miR-199a were 5'-TCAAGAGCAATAACGAAAAATGT-3' (forward) and 5'-GCTGTCAACGATACGCTACGT-3' (reverse). For normalization control, the U6 snRNA was used. The primers used for ROCK1 were 5'-AGGAAG GCGGACATATTAGTCCCT-3' (forward) and 5'-AGACGA
TAGTTGGGTCCCGGC-3' (reverse). The primers used for GAPDH were 5'-AACGTGTCAGTGGTGGACCTG-3' (forward) and 5'-AGTGGGTGTCGCTGTTGAAGT-3' (reverse). The relative expression of each gene was calculated and normalized using the $2^{-\Delta \Delta \mathrm{Ct}}$ method relative to U6 snRNA or GAPDH. PCR amplification was repeated three times for each gene.

\section{Lentivirus (LV) production and infection}

The pLV-has-miR-199a plasmid, together with the control (pLV-miRNA-vector), was obtained from Biosettia Inc. (San Diego, CA, USA). We packaged viruses and infected the cells following standard protocols. We named the packaged lentiviruses as LV-miR-199a and LV-ctrl.

\section{Plasmid construction and luciferase reporter assays}

To carry out luciferase reporter assay, we amplified 3'-UTR segments containing the miR-199a-binding sites of ROCK1 and inserted them into the pGL3-control vector (Promega, Madison, WI, USA). Site-directed mutagenesis of the miR-199a seed sequence in the ROCK1 3'-UTR (Mut) was performed using the QuikChange ${ }^{\mathrm{TM}}$ Site-Directed Mutagenesis Kit (Stratagene, San Diego, CA, USA). Subsequently, LV-miR-199a cells were transfected with wild-type or mutant reporter plasmids, followed by evaluation of the luciferase activity with the Dual-Luciferase Reporter Assay System (Promega) 48 hours later. Full-length ROCK1 cDNA entirely lacking the 3'-UTR was purchased from GeneCopoeia (Rockville, MD, USA) and subcloned into the eukaryotic expression vector pcDNA.3 (Thermo Fisher Scientific).

\section{Oligonucleotide transfection}

siRNA duplex oligonucleotides targeting human Snail mRNA (sense: 5'-GGACACAGGUUCUGAAACA-3' and anti-sense: $5^{\prime}$-CCUGUGUCCAAGACUUUGU-3') and the sequence of si-negative control (si-ctrl) were designed by RiboBio (Guangzhou, China). pcDNA.3-Snail was a kind gift from Dr Ming Li (Sun Yat-Sen University, Guangzhou, China). Oligonucleotide transfection was performed with Lipofectamine 2000 reagent (Thermo Fisher Scientific).

\section{MTT, colony formation and cell invasion assays}

MTT assay was used to analyze cell proliferation. Briefly, $4 \times 10^{3}$ cells were seeded into 96 -well culture plates. Subsequently, the cells were incubated with MTT. Then, the 
culture supernatant was removed, and dimethyl sulfoxide was added to solubilize the crystals. The absorbance value (OD) of each well was measured at $490 \mathrm{~nm}$. Experiments were performed three times.

For colony formation, 24 hours after infection, 300 infected cells were placed in a fresh six-well plate and maintained in DMEM containing 10\% FBS for 2 weeks. Colonies were fixed with methanol and stained with $0.1 \%$ crystal violet in $20 \%$ methanol for 15 minutes. Experiments were performed three times.

To perform invasion assays, 105 cells were added to a Matrigel invasion chamber (BD Biosciences, San Jose, CA, USA), which was present in the insert of a 24-well culture plate. Cells were plated in medium without serum, and medium containing $10 \%$ FBS in the lower chamber served as a chemoattractant. After the cells were incubated for 8 hours at $37^{\circ} \mathrm{C}$ in a $5 \% \mathrm{CO}_{2}$ atmosphere, the insert was washed with PBS, and cells on the top surface of the insert were removed with a cotton swab. Invasive cells located on the lower side of the chamber were stained with crystal violet, air-dried and photographed. Experiments were performed three times.

\section{Apoptosis assay}

Apoptosis was measured using an Annexin V/fluorescein isothiocyanate apoptosis detection kit (Keygen, Nanjing, China) according to the manufacturer's instructions. Data acquisition and analyses were done on a Becton Dickinson (Franklin Lakes, NJ, USA) FACSCalibur using CellQuest software (Becton Dickinson). Each experiment was performed in triplicate.

\section{Western blot and Northern blot analysis}

Cells were lysed in RIPA buffer in the presence of proteinase inhibitor cocktail. Then, the total protein extracts were separated and transferred to polyvinylidene fluoride membranes. After blocking with 5\% nonfat milk, the membranes were incubated with primary antibodies against ROCK1 and GAPDH (Santa Cruz Biotechnology Inc., Dallas, TX, USA) overnight at $4^{\circ} \mathrm{C}$ and then blotted for 1 hour at room temperature with an appropriate secondary antibody, followed by enhanced chemiluminescence detection reagents (Amersham Pharmacia Biotech, Little Chalfont, UK).

Small RNA Northern blot analysis was performed as previously described. ${ }^{23}$ Band intensities were measured using Quantity One image analysis software (Bio-Rad, Hercules, CA, USA). The RNA Northern Blot Assay Kit for miR-199a was purchased from BioCat GmbH (Heidelberg, Germany) (Cat No MP-0080-SO).

\section{Immunofluorescence assay}

Cells were fixed in $4 \%$ formaldehyde, and then permeabilized with $0.1 \%$ Triton X-100. After blocking with BSA, these cells were incubated with tetramethylrhodamine B isothiocyanate-labeled phalloidin and observed under a fluorescence microscope.

\section{Chromatin immunoprecipitation PCR}

Using Chromatin Immunoprecipitation Kit (Merck Millipore, Billerica, MA, USA), we immunoprecipitated DNA-protein complexes from A498 cells and transfected them with Snail cDNA. qRT-PCR analysis was performed to amplify the miR199a promoter region from the precipitated DNA. The primers used in the PCR were 5'-GGAAAUCAACCCUGAAUGC-3' (sense) and 5'-CCCAUGCCAAAGACAGGGU-3' (antisense). Each experiment was performed in triplicate.

\section{In vivo tumorigenesis}

A498 cells $\left(4 \times 10^{6}\right)$ were transplanted into the flanks of nude mice (six mice for each group). We calculated the tumor volume using the formula: volume $\left(\mathrm{mm}^{3}\right)=L \times W^{2} / 2$ (length, $L$, $\mathrm{mm}$; width, $W, \mathrm{~mm}$ ). All animal experiments were performed according to the Institutional Animal Care and Use Committee (IACUC)-approved animal protocols and all animal experimental procedures were approved by the Experimental Animal Ethical Committee of Jining Medical University.

\section{Statistical analysis}

All values are presented as the mean \pm standard error of the mean (SEM). A one-way ANOVA or two-tailed Student's $t$-test was used for comparisons between groups. KaplanMeier method was used to perform the survival analysis. SPSS software package (SPSS Standard version 13.0; SPSS Inc.) was used for statistical analysis, and $P<0.05$ was considered statistically significant.

\section{Results \\ Lower levels of miR-199a are frequently detected in ccRCC tissues and associated with poor prognostic phenotype}

The expression levels of miR-199a were first examined by realtime PCR in 26 pairs of ccRCC and adjacent non-neoplastic renal tissues. The results showed that miR-199a was significantly downregulated in ccRCC tissues compared with adjacent non-neoplastic renal tissues (Figure 1A, $P<0.05$ ). Furthermore, the relationship between miR-199a expression and overall survival was determined through Kaplan-Meier 

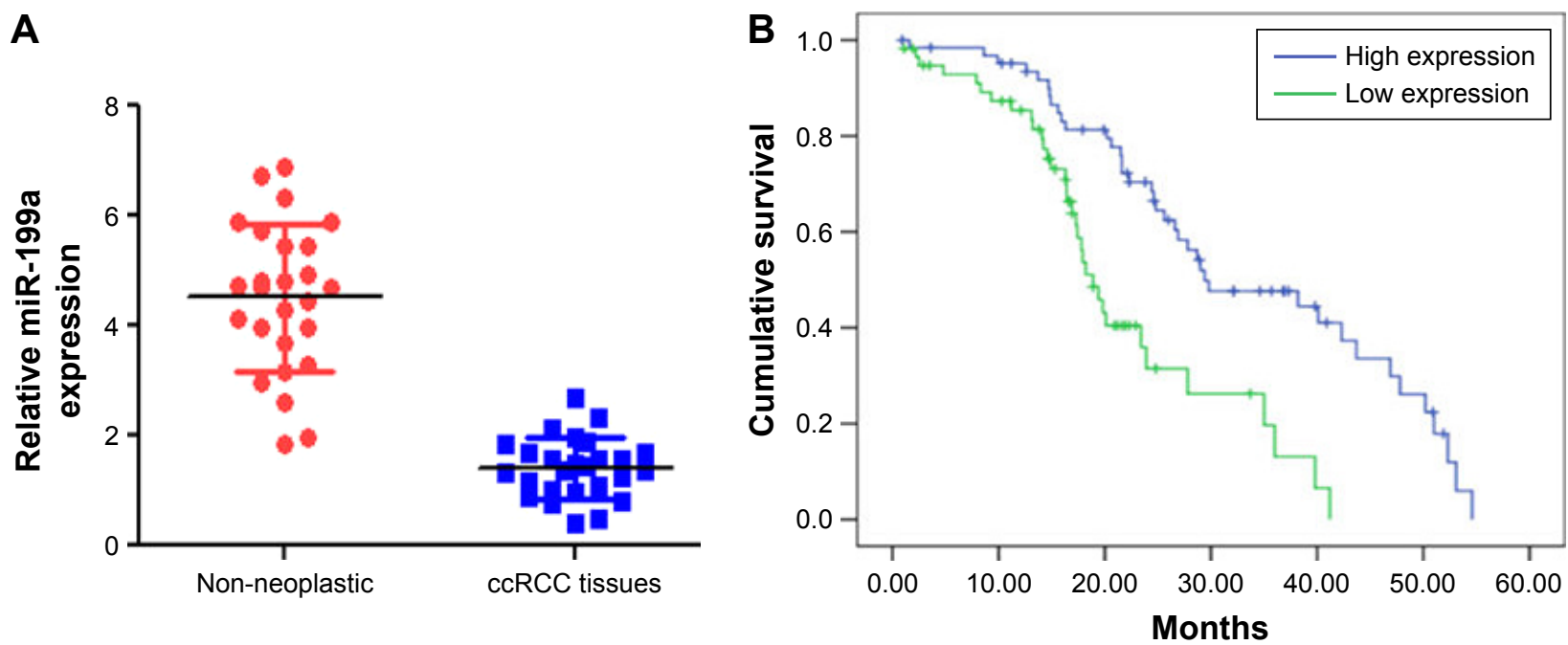

Figure I Lower levels of miR-199a were frequently detected in ccRCC tissues and associated with poor prognostic phenotype. (A) The expression of miR-199a was significantly downregulated in ccRCC tissues compared with adjacent non-neoplastic renal tissues. (B) ccRCC patients with lower-than-median levels of miR-I99a expression had poorer survival in contrast to those with higher-than-median levels of miR-199a expression.

Abbreviation: ccRCC, clear cell renal cell carcinoma.

survival curve analysis with a log-rank comparison of 98 ccRCC patients. We observed that ccRCC patients with lower-than-median levels of miR-199a expression had poorer survival in contrast to those with higher-than-median levels of miR-199a expression, who had longer survival times (Figure 1B, $P<0.05$ ).

\section{miR-199a targets ROCKI in ccRCC cells}

It is generally accepted that miRNAs exert their function by regulating expression of their downstream target gene(s). miRanda and TargetScan algorithms were used to search for target genes of miR-199a. Our analysis revealed that ROCK1 was one of the potential targets of miR-199a. Indeed, the 3'-UTR of ROCK1 mRNA contains a complementary site for the seed region of miR-199a (Figure 2A). Subsequently, we subcloned ROCK1 wild-type (WT) or mutant (Mut) 3'-UTR (Figure 2A) into a luciferase reporter vector. Then, the A498 cells were co-transfected with miR-199a or scrambled control and ROCK1 wild-type or mutant 3'-UTR. It was found that miR-199a significantly inhibited the luciferase activity of the ROCK1 wild-type 3'-UTR but not that of the Mut 3'-UTR' in A498 cells (Figure 2B, $P<0.05$ ). Furthermore, overexpression of miR-199a significantly downregulated ROCK1 mRNA and protein levels in A498 cells (Figure 2C and D).

\section{Exogenetic overexpression of miR-199a} suppresses proliferation and invasion but induces apoptosis in ccRCC cells

Given the fact that ROCK1 is involved in the regulation of many key cellular functions associated with malignancy, ${ }^{24}$ we asked whether restoration of miR-199a may suppress proliferation and invasion in ccRCC cells. As shown in Figure 3A and B, miR-199a overexpression significantly inhibited ccRCC cell proliferation and colony formation. However, ROCK1-overexpressing vector (pcDNA.3ROCK1) can counteract this effect.

Next, the effect of miR-199a on invasive capacity of ccRCC cells was characterized by Matrigel invasion assays. The results demonstrated that exogenetic overexpression of miR-199a markedly reduced invasiveness of ccRCC cells, while ROCK1 restoration could partially block these effects (Figure 3C).

We further examined whether restoration of miR-199a may affect apoptosis in ccRCC cells. The rate of cellular apoptosis in A498 cells was examined using flow cytometric analysis with Annexin V staining. As shown in Figure 3D, miR-199a overexpression induced apoptosis in A498 cells, while exogenetic overexpression of ROCK1 blocked the miR-199a effect on apoptosis.

It was found that stress fiber formation was suppressed in miR-199a-treated cells. In addition, it was observed that the filopodia and lamellipodia were reduced on the cell surfaces of the miR-199a-treated cells. Interestingly, exogenetic overexpression of ROCK1 partly blocked these effects induced by miR-199a (Figure S1A and B).

\section{miR-199a inhibits tumor growth in vivo}

To further confirm the suppressive roles of miR-199a in tumor growth in vivo, we initially infected A498 cells with lentiviral vectors expressing miR-199a stably (LV-miR-199a) or the 
A

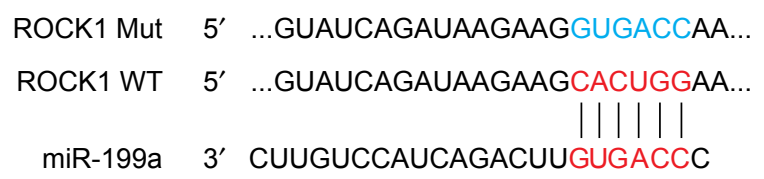

C

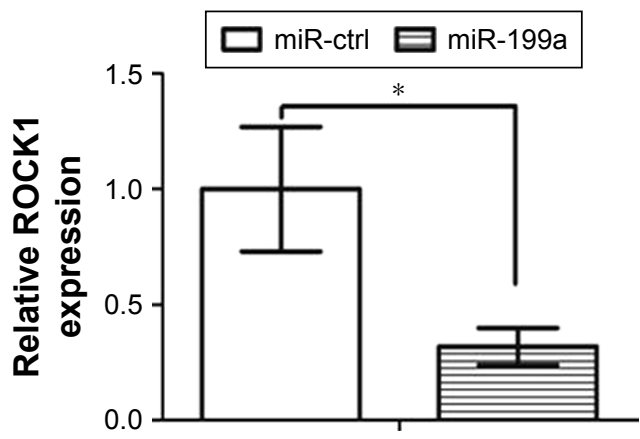

B

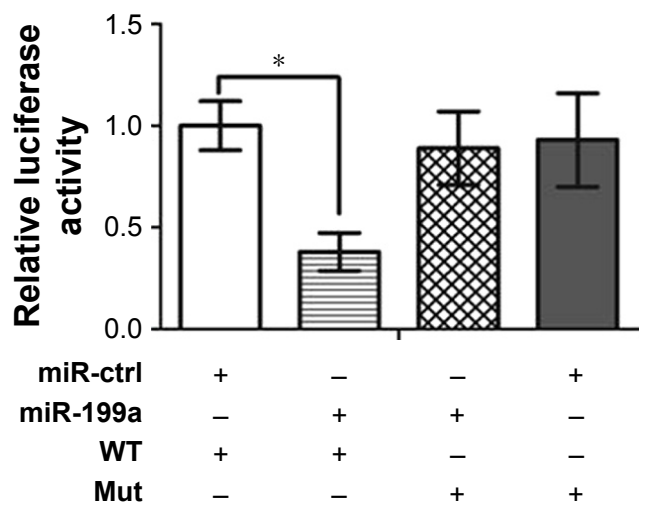

D

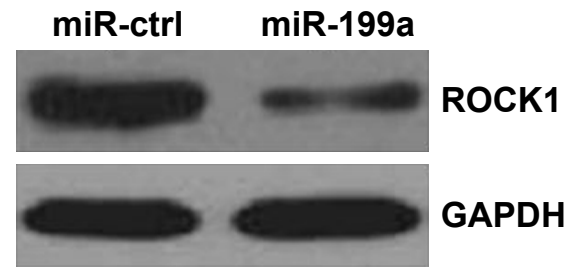

Figure 2 ROCKI was a direct target of miR-199a in A498 cells.

Notes: (A) Sequence alignment of miR-199a and ROCKI 3'-UTR, which contains one predicted miR-199a-binding site. The seed-recognizing sites in the ROCKI 3'-UTR is indicated in red, while the ROCKI Mut 3'-UTR is indicated in blue. (B) Luciferase assay in A498 cells co-transfected with miR-199a and luciferase reporter containing ROCKI WT or a Mut 3'-UTR. (C) miR-199a transfection affects ROCKI mRNA levels. $* P<0.05$. (D) miR-199a transfection affects ROCKI protein levels.

Abbreviations: ctrl, control; Mut, mutant; ROCKI, Rho-associated coiled coil-containing protein kinase I; WT, wildtype.

negative control (LV-ctrl). Stable transfection of miR-199a into A498 cells resulted in decreased growth and tumor weight (Figure 4A) of subcutaneous xenograft tumors in nude mice, when compared with those stably transfected with empty vector. Furthermore, immunohistochemical staining demonstrated that the tumors originating from LV-miR-199a cells had decreased expression of Ki-67 and ROCK1, compared with that from LV-ctrl cells (Figure 4B). These results showed that miR-199a can inhibit tumor growth in vivo.

\section{miR-199a levels are inversely correlated with mRNA expression of ROCKI in cCRCC tissues}

We further examined the miR-199a levels and the mRNA expression of ROCK1 in 39 ccRCC tissues. We found a significant inverse correlation between the levels of miR199a and mRNA expression of ROCK1 in these tissues (Figure 4C).

\section{Snail regulates ROCKI expression by modulating miR-199a}

Like protein-coding mRNAs, miRNAs are also transcribed from their genes in genomic DNA. Therefore, the expression of each miRNA is driven by a promoter and regulated by transcription factors. ${ }^{25} \mathrm{We}$ first found a conserved DNA region upstream of pri-miR-199a gene, which was reported to contain the pri-miR-199a gene promoter. ${ }^{26}$ With the use of Consite program (http://consite.genereg. net/), we found there were binding sites of transcription factor Snail in the DNA region (Figure 5A). The DNA region was cloned into pGL3-basic vector (Promega), and this vector was named pGL3-miR-199a pro. The luciferase assay showed that this DNA region contains the pri-miR-199a promoter (Figure 5B). However, when pGL3-miR-199a pro vector was co-transfected with Snail expression vector (Snail), the luciferase activity was efficiently repressed (Figure 5C). Moreover, qRT-PCR assay showed that Snail could inhibit the expression level of miR-199a, which was consistent with the Northern blot assay (Figure 5D). We further correlated the expression level of miR-199a with the Snail mRNA level in the same ccRCC tissues. As displayed in Figure 5E, a significant inverse correlation was found between the expression level of miR-199a and Snail mRNA. Taken together, these results demonstrate that Snail is an upstream regulator of miR-199a expression. 
A

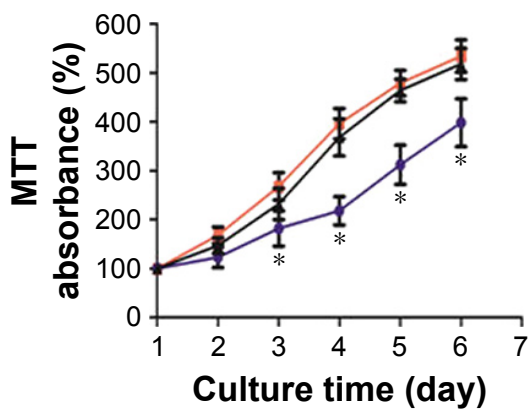

+- miR-ctrl $\rightarrow$ miR-199a
+ miR-199a + pcDNA.3 ROCK1
B

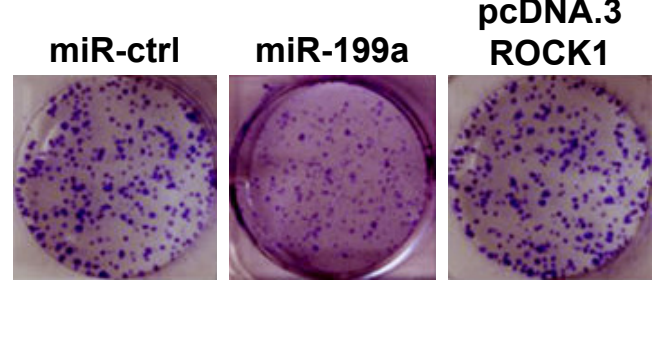

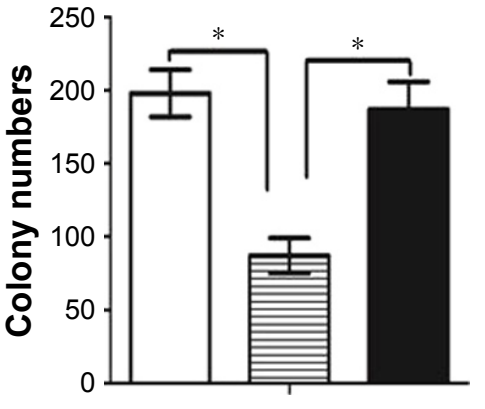

miR-ctrl 日miR-199a miR-199a + pcDNA.3 ROCK1

C
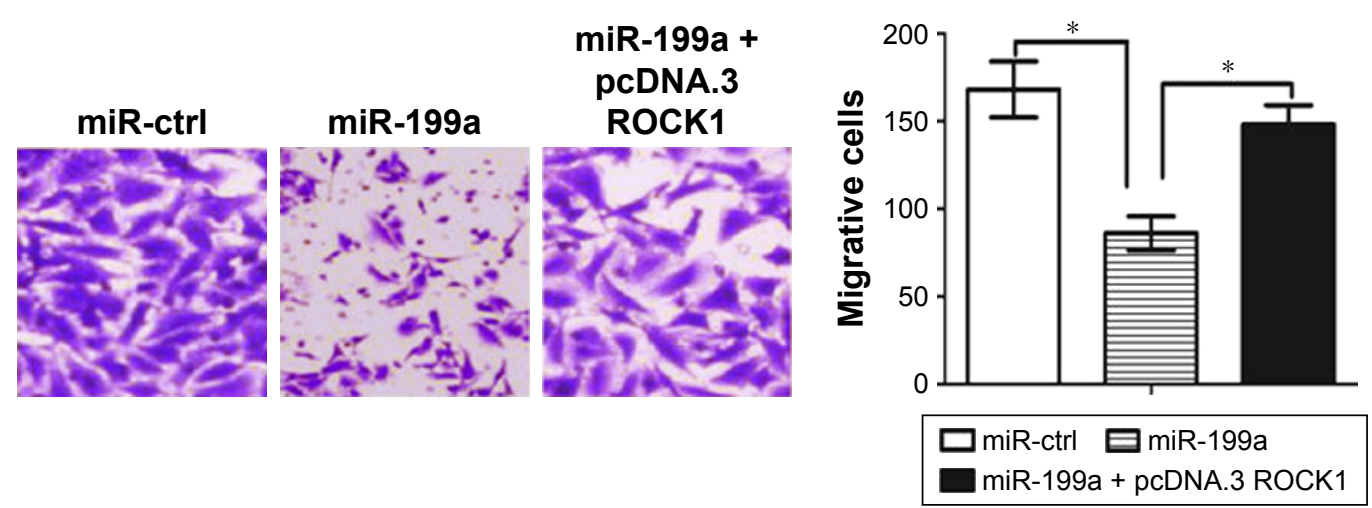

D
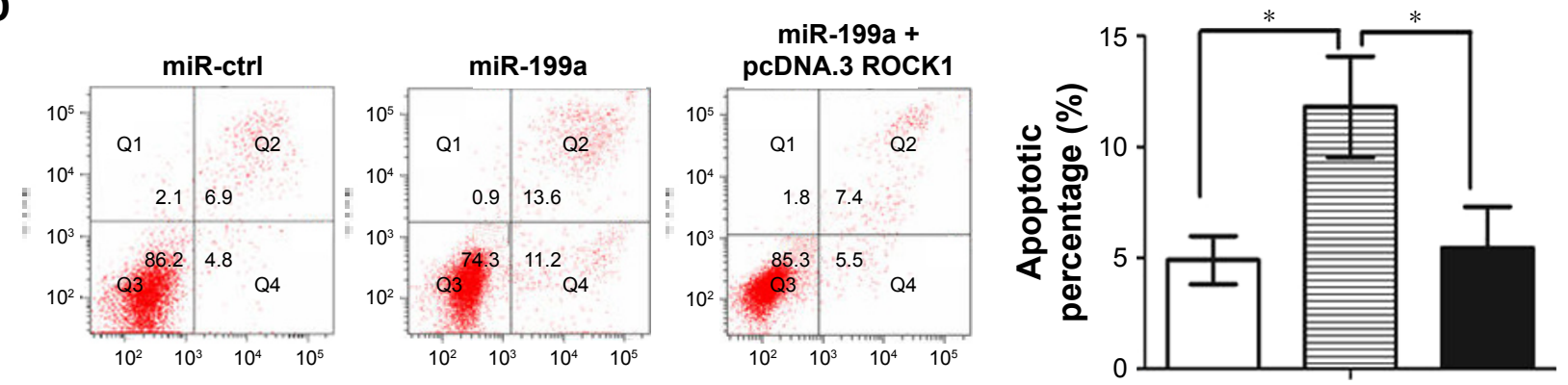

miR-ctrl $\boxminus m i R-199 a$

miR-199a + pcDNA.3 ROCK1

Figure 3 Restoration of miR-199a suppressed proliferation and colony formation, but induced apoptosis in ccRCC cells.

Notes: (A) Restoration of miR-199a inhibited A498 cell proliferation. (B) Overexpression of miR-199a impeded colony-forming ability of A498 cells. (C) Exogenetic overexpression of miR-199a markedly reduced invasiveness of A498 cells, while ROCKI restoration could partially block these effects (magnification, $\times 400$ ). (D) miR-199a overexpression induced apoptosis in A498 cells, while exogenetic overexpression of ROCKI blocked the miR-I99a effect on apoptosis. $* P<0.05$.

Abbreviations: ccRCC, clear cell renal cell carcinoma; ctrl, control; ROCKI, Rho-associated coiled coil-containing protein kinase I.

\section{Discussion}

In this study, we focused on miR-199a and investigated its tumor-suppressive function and how miR-199a-mediated cancer pathways contributed to ccRCC progression. We observed that miR-199a expression was decreased in human ccRCC tissues compared with matching adjacent normal tissues and that miR-199a suppressed tumor growth both in vitro and in vivo.
miRNAs not only contribute to diverse biological processes but are also implicated in the progression and metastasis of human cancers..$^{27}$ Deregulation of miRNAs is associated with several diseases including cancer. ${ }^{18}$ Insights into the roles of miRNAs in cancer have made them targets for novel therapeutic approaches. Recently, several miRNAtargeted therapeutics have reached clinical development. ${ }^{28}$ Consistent with the above findings, we found that restoration 
A
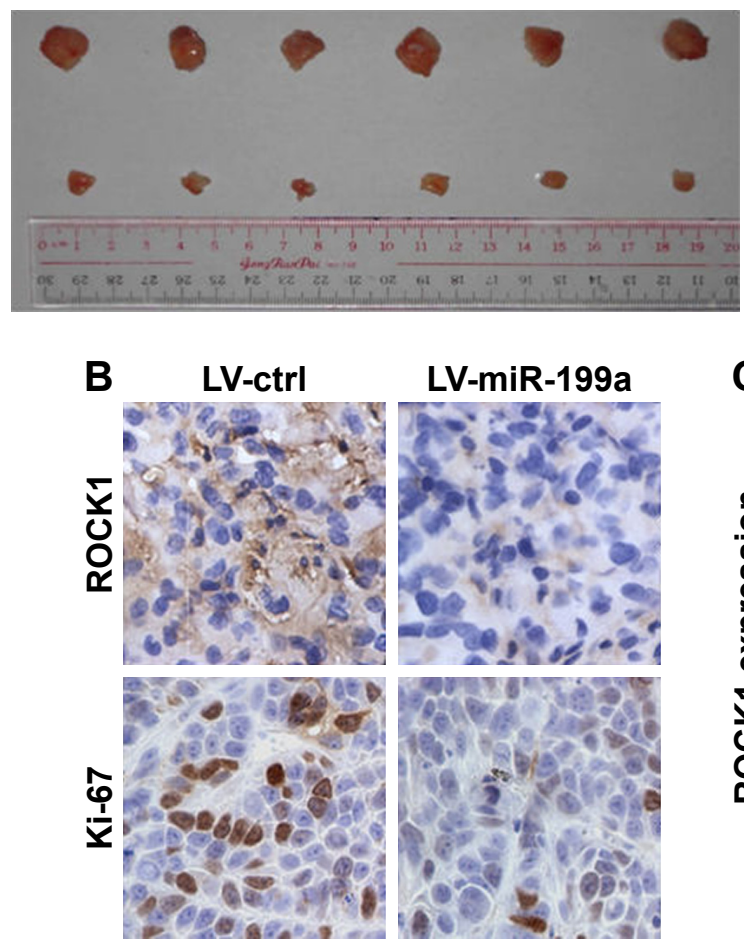
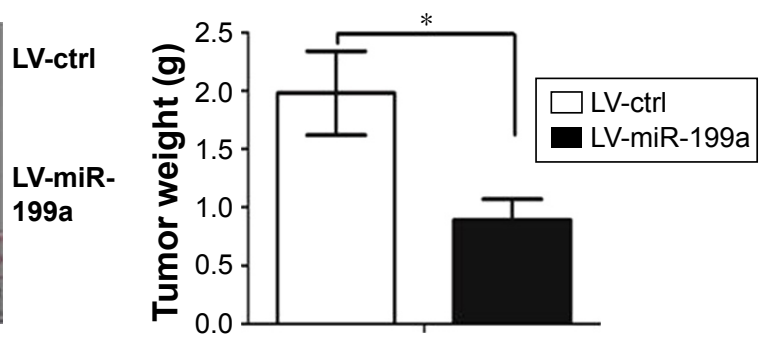

C

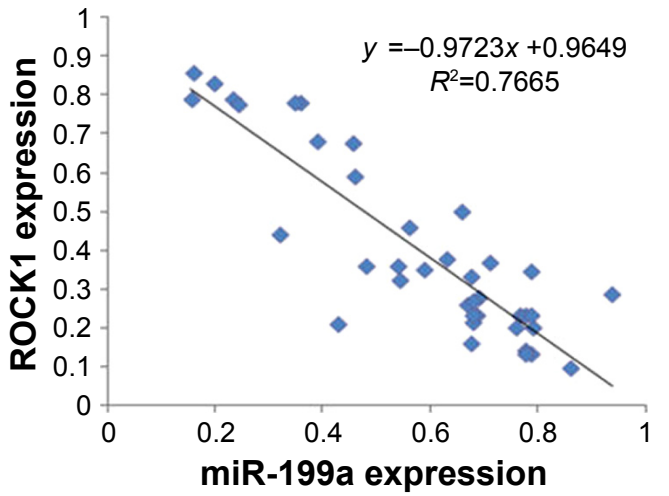

Figure 4 miR-199a inhibited tumor growth in vivo.

Notes: (A) Stable transfection of miR-199a into A498 cells resulted in decreased growth and tumor weight. $* P<0.05$. (B) Immunohistochemical staining demonstrated that the tumors originating from LV-miR-199a cells had decreased expression of Ki-67 and ROCKI, compared with that from LV-ctrl cells (magnification, $\times 400$ ). (C) We found an inverse correlation between the levels of miR-199a and mRNA expression of ROCKI.

Abbreviations: ctrl, control; LV, lentiviral vectors; ROCKI, Rho-associated coiled coil-containing protein kinase I.

of miR-199a could suppress proliferation and invasion, but induce apoptosis in ccRCC cells. Using dual-luciferase reporter assay and Western blot analysis, we further confirmed that ROCK1 was a direct target of miR-199a.
ROCK1 is a serine/threonine kinase that belongs to the Rho family of GTPase proteins that facilitate the reorganization of the actin cytoskeleton, a pivotal event during cell motion and invasion. ${ }^{29}$ ROCK1 is overexpressed in

\section{A}

\section{Putative transcription factor-binding sites} found along miR-199a
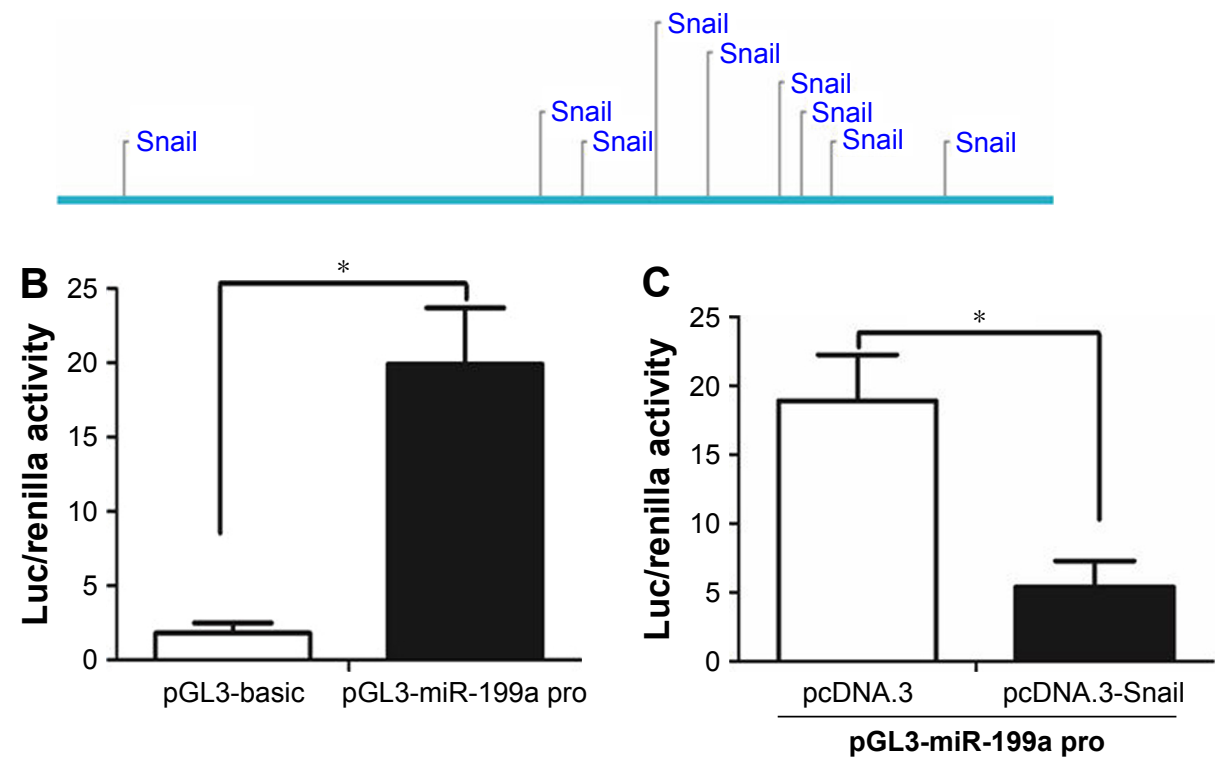

Figure 5 (Continued) 

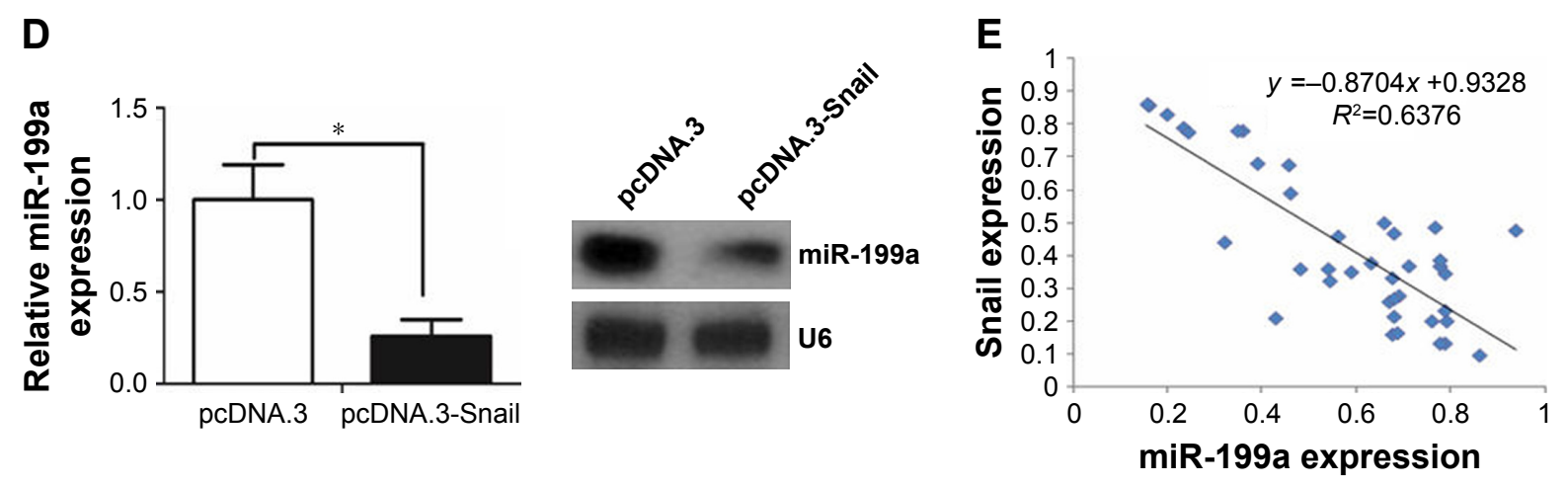

Figure 5 Snail regulated miR-199a expression.

Notes: (A) The putative binding sites of transcription factor Snail in the DNA region of miR-199a. (B) The pGL3-miR-199a pro vector was capable of directing luciferase expression. (C) Luciferase activity was efficiently repressed when PGL3-miR-199a pro vector was co-transfected with pcDNA.3-Snail. (D) The qRT-PCR and Northern blot analysis showed that Snail decreased miR-199a expression. (E) Inverse correlation between miR-199a expression and Snail mRNA level in primary ccRCC tissues. $* P<0.05$. Abbreviations: Luc, luciferase; qRT-PCR, quantitative real-time PCR; ccRCC, clear cell renal cell carcinoma.

various cancers, including ccRCC. ${ }^{24,30}$ ROCK1 mediates multiple pathways regulating cell proliferation, invasion and apoptosis. ${ }^{31,32}$ Our findings showed that miR-199a exerts its tumor-suppressive function in ccRCC cells partially through ROCK1. Importantly, we further found that miR-199a levels are inversely correlated with mRNA expression of ROCK1 in ccRCC tissues. It is worth noting that some other target sites of miR-199a (eg, TGF beta, ${ }^{33}$ c-met $^{34}$ and mTOR $^{35}$ ) may also mediate its effect on ccRCC. Further investigation is needed to clarify the role of miR-199a in ccRCC.

Snail is a DNA-binding zinc finger protein and functions as a transcriptional repressor. It has been reported that Snail is associated with lymph node metastasis, tumor relapse and prognosis. In the current study, we revealed that Snail repressed miR-199a expression in ccRCC cells, and finally led to the activation of ROCK1. Restoration of miR-199a could partly counteract the effect of Snail on ROCK1 expression. We further found a distinct inverse correlation between the expression level of Snail mRNA and miR-199a in ccRCC tissue samples. Some other transcription factors, such as c-myc, c-jun and NF-kappa, are predicted to regulate miR-199a expression. Further investigation is warranted to explore the interaction among these transcription factors and miR-199a.

Taken together, this study found that miR-199a acts as a growth-suppressive miRNA in human ccRCC both in vitro and in vivo. As miR-199a is downregulated in ccRCC, reintroduction of this mature miRNA into the tumor tissue could provide a therapeutic strategy. Although miRNA-based therapeutics are still in their infancy, our findings on miR-199a are encouraging and suggest that this miRNA could be a potential target for the treatment of ccRCC in future.

\section{Acknowledgment}

This work was supported by a grant from the Natural Science Foundation of Guangdong Province, China (number 2015A030310111).

\section{Disclosure}

The authors report no conflicts of interest in this work.

\section{References}

1. McLaughlin JK, Lipworth L, Tarone RE. Epidemiologic aspects of renal cell carcinoma. Semin Oncol. 2006;33(5):527-533.

2. Cheng L, Zhang S, MacLennan GT, Lopez-Beltran A, Montironi R. Molecular and cytogenetic insights into the pathogenesis, classification, differential diagnosis, and prognosis of renal epithelial neoplasms. Hum Pathol. 2009;40(1):10-29.

3. Ljungberg B, Bensalah $\mathrm{K}$, Canfield $\mathrm{S}$, et al. EAU guidelines on renal cell carcinoma: 2014 update. Eur Urol. 2015;67(5):913-924.

4. Bex A, Jonasch E, Kirkali Z, et al. Integrating surgery with targeted therapies for renal cell carcinoma: current evidence and ongoing trials. Eur Urol. 2010;58(6):819-828.

5. Thillai K, Allan S, Powles T, Rudman S, Chowdhury S. Neoadjuvant and adjuvant treatment of renal cell carcinoma. Expert Rev Anticancer Ther. 2012;12(6):765-776.

6. Yang JC, Haworth L, Sherry RM, et al. A randomized trial of bevacizumab, an anti-vascular endothelial growth factor antibody, for metastatic renal cancer. $N$ Engl J Med. 2003;349(5):427-434.

7. Motzer RJ, Hutson TE, Tomczak P, et al. Sunitinib versus interferon alfa in metastatic renal-cell carcinoma. $N$ Engl J Med. 2007;356(2):115-124.

8. Srinivasan R, Ricketts CJ, Sourbier C, Linehan WM. New strategies in renal cell carcinoma: targeting the genetic and metabolic basis of disease. Clin Cancer Res. 2015;21(1):10-17.

9. Johndrow JE, Magie CR, Parkhurst SM. Rho GTPase function in flies: insights from a developmental and organismal perspective. Biochem Cell Biol. 2004;82(6):643-657.

10. Kumper S, Mardakheh FK, McCarthy A, et al. Rho-associated kinase (ROCK) function is essential for cell cycle progression, senescence and tumorigenesis. Elife. 2016;5(1):e12994.

11. Liu Y, Zhou H, Zhu R, et al. SPSB3 targets SNAIL for degradation in GSK- $3 \beta$ phosphorylation-dependent manner and regulates metastasis. Oncogene. Epub 2017 Oct 23. 
12. Meng FD, Li Y, Tian X, et al. Synergistic effects of snail and quercetin on renal cell carcinoma Caki-2 by altering AKT/mTOR/ERK1/2 signaling pathways. Int J Clin Exp Pathol. 2015;8(6):6157-6168.

13. Kumar AS, Jagadeeshan S, Pitani RS, et al. Snail-modulated microRNA 493 forms a negative feedback loop with the insulin-like growth factor 1 receptor pathway and blocks tumorigenesis. Mol Cell Biol. 2017; 37(6):e00510-e00516.

14. Bartel DP. MicroRNAs: genomics, biogenesis, mechanism, and function. Cell. 2004;116(2):281-297.

15. He L, Hannon GJ. MicroRNAs: small RNAs with a big role in gene regulation. Nat Rev Genet. 2004;5(7):522-531.

16. Bartel DP. MicroRNAs: target recognition and regulatory functions. Cell. 2009;136(2):215-233.

17. Calin GA, Croce CM. MicroRNA signatures in human cancers. Nat Rev Cancer. 2006;6(11):857-866.

18. Esquela-Kerscher A, Slack FJ. Oncomirs - microRNAs with a role in cancer. Nat Rev Cancer. 2006;6(4):259-269.

19. Liu GT, Huang YL, Tzeng HE, Tsai CH, Wang SW, Tang CH. CCL5 promotes vascular endothelial growth factor expression and induces angiogenesis by down-regulating miR-199a in human chondrosarcoma cells. Cancer Lett. 2015;357(2):476-487.

20. Song J, Gao L, Yang G, et al. MiR-199a regulates cell proliferation and survival by targeting FZD7. PLoS One. 2014;9(10):e110074.

21. Joshi HP, Subramanian IV, Schnettler EK, et al. Dynamin 2 along with microRNA-199a reciprocally regulate hypoxia-inducible factors and ovarian cancer metastasis. Proc Natl Acad Sci U S A. 2014;111(14): 5331-5336.

22. He XJ, Ma YY, Yu S, et al. Up-regulated miR-199a-5p in gastric cancer functions as an oncogene and targets klotho. BMC Cancer. 2014;14(3):218.

23. McClure LV, Lin YT, Sullivan CS. Detection of viral microRNAs by Northern blot analysis. Methods Mol Biol. 2011;721:153-171.

24. Schofield AV, Bernard O. Rho-associated coiled-coil kinase (ROCK) signaling and disease. Crit Rev Biochem Mol Biol. 2013;48(4):301-316.
25. Rodriguez A, Griffiths-Jones S, Ashurst JL, Bradley A. Identification of mammalian microRNA host genes and transcription units. Genome Res. 2004;14(10A):1902-1910.

26. Sakurai K, Furukawa C, Haraguchi T, et al. MicroRNAs miR-199a-5p and $-3 p$ target the Brm subunit of SWI/SNF to generate a doublenegative feedback loop in a variety of human cancers. Cancer Res. 2011;71(5):1680-1689.

27. Wang D, Qiu C, Zhang H, Wang J, Cui Q, Yin Y. Human microRNA oncogenes and tumor suppressors show significantly different biological patterns: from functions to targets. PLoS One. 2010;5(9):pii:e13067.

28. Chen CZ. MicroRNAs as oncogenes and tumor suppressors. $N$ Engl J Med. 2005;353(17):1768-1771.

29. Narumiya S, Tanji M, Ishizaki T. Rho signaling, ROCK and mDia1, in transformation, metastasis and invasion. Cancer Metastasis Rev. 2009;28(1-2):65-76.

30. Abe H, Kamai T, Tsujii T, et al. Possible role of the RhoC/ROCK pathway in progression of clear cell renal cell carcinoma. Biomed Res. 2008;29(3):155-161.

31. Majid S, Dar AA, Saini S, et al. MicroRNA-1280 inhibits invasion and metastasis by targeting ROCK1 in bladder cancer. PLoS One. 2012;7(10):e46743.

32. Vemula S, Shi J, Mali RS, et al. ROCK1 functions as a critical regulator of stress erythropoiesis and survival by regulating p53. Blood. 2012;120(14):2868-2878

33. Wala SJ, Karamchandani JR, Saleeb R, et al. An integrated genomic analysis of papillary renal cell carcinoma type 1 uncovers the role of focal adhesion and extracellular matrix pathways. Mol Oncol. 2015; 9(8):1667-1677.

34. Giglio S, Vecchione A. c-Met and miRs in Cancer. Biomedicines. 2015; 3(1):32-44.

35. Aguiari G. MicroRNAs in clear cell renal cell carcinoma: biological functions and applications. J Kidney Cancer VHL. 2015;2(4):140-152. 


\section{Supplementary material}

A

miR-ctrl

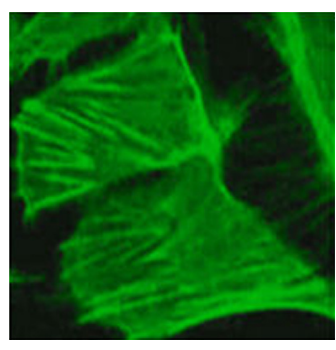

B

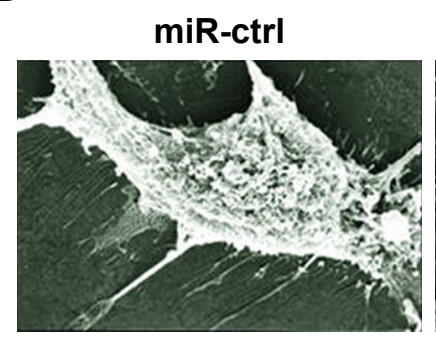

$\operatorname{miR}-199 a$

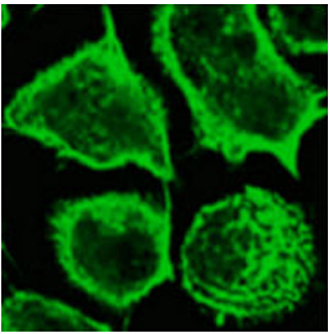

miR-199a

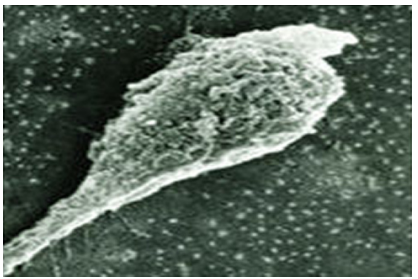

$\operatorname{miR}-199 a+$ PCDNA.3 ROCK1

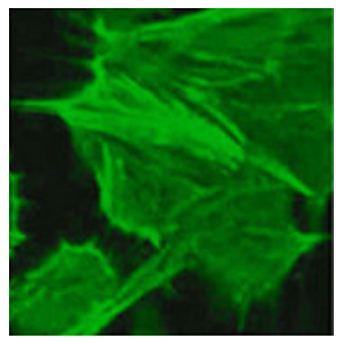

miR-199a + PcDNA.3 ROCK1

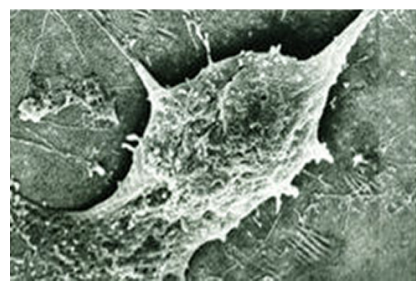

Figure SI (A) Ectopic expression of miR-199a disrupted the stress fiber network. Stress fibers (polymerized actin) and actin filaments were visualized by phalloidin staining. (B) The cell morphology alteration among miR-ctrl, miR-199a and miR-199a + ROCKI groups observed under scanning electron microscope. Note: Magnification, $\times 1000$.

Abbreviations: ctrl, control; ROCKI, Rho-associated coiled coil-containing protein kinase I.

\section{Publish your work in this journal}

OncoTargets and Therapy is an international, peer-reviewed, open access journal focusing on the pathological basis of all cancers, potential targets for therapy and treatment protocols employed to improve the management of cancer patients. The journal also focuses on the impact of management programs and new therapeutic agents and protocols on patient perspectives such as quality of life, adherence and satisfaction. The manuscript management system is completely online and includes a very quick and fair peer-review system, which is all easy to use. Visit http://www.dovepress.com/testimonials.php to read real quotes from published authors. 